\title{
Shorter communication
}

\section{Experiential avoidance and aversive visual images: Response delays and event-related potentials on a simple matching task}

\author{
Andy Cochrane ${ }^{\mathrm{a}, *}$, Dermot Barnes-Holmes ${ }^{\mathrm{a}}$, Yvonne Barnes-Holmes $^{\mathrm{a}}$, \\ Ian Stewart ${ }^{\mathrm{b}}$, Carmen Luciano ${ }^{\mathrm{c}}$ \\ ${ }^{a}$ Department of Psychology, National University of Ireland Maynooth, Maynooth, Co. Kildare, Ireland \\ ${ }^{\mathrm{b}}$ National University of Ireland, Galway, Ireland \\ ${ }^{\mathrm{c}}$ University of Almeria, Almeria, Spain
}

Received 20 January 2006; received in revised form 12 May 2006; accepted 19 May 2006

\begin{abstract}
In Experiment 1, participants high $(n=15)$ or low in avoidance $(n=14)$, as measured by the Acceptance and Action Questionnaire, completed a simple matching task that required them to choose whether or not to look at an aversive visual image. Only the high-avoidance participants took longer to emit a correct response that produced an aversive rather than a neutral picture. Additionally, the high-avoiders reported greater levels of anxiety following the experiment even though they rated the aversive images as less unpleasant and less emotionally arousing than their low-avoidant counterparts. In Experiment 2, three groups, representing high-, mid- and low-avoidance $(n=6$ in each) repeated the matching task with the additional recording of event-related potentials (ERPs). The findings of Experiment 1 were replicated in terms of reaction times and subjective ratings. The ERPs confirmed that the participants attended to the content of the images and differentiated between the aversive and neutral image types. The ERPs also showed significantly greater negativity for electrodes over the left hemisphere relative to the midline for only the high-experiential avoidance (EA) group. Given the left hemisphere dominance for language, the data suggest that the high-EA group engaged in verbal strategies to regulate their emotional responses.
\end{abstract}

(C) 2006 Elsevier Ltd. All rights reserved.

Keywords: Experiential avoidance; Event-related potentials; International affective picture system; Acceptance and action questionnaire

\section{Introduction}

According to Hayes, Strosahl, \& Wilson (1999) an individual pre-disposed towards experiential avoidance (high-EA) may attempt to suppress any unwanted thoughts, feelings and bodily sensations, or actively avoid

*Corresponding author. Tel.: + 35317084765 ; fax: + 35317084767 .

E-mail address: cochrane.andy@gmail.com (A. Cochrane). 
any situations that may elicit these unwanted private events. Recent studies have sought to determine if individuals predisposed towards high-EA, as measured by the Acceptance and Action Questionnaire (AAQ; Hayes et al., 2004), experience greater levels of anxiety and affective distress, relative to low-EA individuals, when exposed to experimentally induced stressors.

These studies have employed physically aversive stimuli (e.g., inhalations of $\mathrm{CO}_{2}$ : Feldner, Zvolensky, Eifert, \& Spira, 2003, and cold-presser tasks: Feldner et al., 2006) and thus it could be argued that the results may not generalise to non-physical stressors, such as emotionally challenging visual material. One recent study, however, has addressed this issue (Sloan, 2004). The participants were shown a series of six brief film clips that were intended to elicit pleasant, unpleasant or neutral emotional states. Consistent with the findings of Feldner et al. (2003) the high-EA group reported greater negative affect following two of the three unpleasant film clips (fear, disgust) compared to the low-EA group. In contrast to these self-reports, the highEA group responded to the fear and disgust film clips with decreased heart rates relative to the low-EA group; neutral film clips produced the opposite effect (i.e., elevated rates in the former group). Sloan suggested that these response patterns might reflect ongoing attempts by the high-EA individuals to regulate their internal experiences leading to greater levels of arousal during resting or neutral periods.

Experiment 1 of the current study sought to extend the Sloan (2004) research by using a computerised procedure that required overt behavioural responding by participants, which provided them with control over the presentation of the visual material (i.e., aversive or neutral pictures). Two measures of trial-by-trial avoidance were recorded; type of image selected and reaction times. Avoiding an aversive stimulus by deliberately selecting the neutral image was associated with negative feedback (i.e. participants were told they had made the 'wrong' choice). Recent findings suggest that avoidant individuals may be more sensitive to potential negative social consequences within an experiment (McAuliffe, 2004), and it was predicted that this may function to override avoidance of the aversive images. In contrast, the time taken to select an aversive versus a neutral image did not lead to any negative feedback, and thus it was predicted that reaction time would prove to be the more robust measure of avoidance in the current study. In short, there should be no difference between high- and low-EA groups on the image-selected measure, but the former group should show longer average reaction times when selecting an aversive image. Experiment 2 sought to replicate and extend Experiment 1 and incorporated electroencephalograms (EEG) as a measure of emotional processing.

\section{Experiment 1}

\section{Method}

\section{Participants}

Participants were selected on the basis of their scores on the 8-item AAQ (note, this version of the AAQ scores in the opposite direction to the original AAQ). Using the same criteria as Feldner et al. (2003), 29 highEA participants were identified from a pool of undergraduates $(N=144) ; 15$ agreed to participate $(9$ female; $\mathrm{M}$ age $=22.6 ; \mathrm{M} \mathrm{AAQ}=32.28 \mathrm{SD}=2.614)$. Fourteen individuals were recruited for the low-EA group (11 female; $\mathrm{M}$ age $=23.29$ years, $\mathrm{M} \mathrm{AAQ}=47.86 \mathrm{SD} 2.57$ ).

\section{Measures}

Questionnaires. Experiential avoidance: The 8-item AAQ was developed from the longer tool (Hayes et al., 2004) and loads onto a single factor $(\alpha=0.77$; Bond et al., personal communication, 10th September, 2003).

Personality measure: The EPQ-R short scale (Eysenck \& Eysenck, 1991) consists of four subscales of 12 items each: Extroversion, Neuroticism, Psychoticism and the Lie Scale. The short scale has good internal consistency with an $\alpha$-coefficient varying from 0.67 to 0.84 in previous Irish studies (Van Hermert, van de Vijver, Poortinga, \& Georgas, 2002).

Pre- and post-experimental anxiety-related measure: The state questionnaire of the Spielberger State-Trait Anxiety Inventory Form-Y (STAI-S, Spielberger, 1983) was employed, primarily to assess any change in affect from pre- to post-experiment. 
Stimulus materials. Seventy-two colour slides were chosen from the International Affective Picture System (IAPS; Lang, Bradley, \& Cuthbert, 1999), depicting 36 unpleasant, aversive events (scenes of violence and bodily mutilations) and 36 neutral events (household objects, neutral faces, and modern buildings). ${ }^{1}$

Subjective measures: The participants were asked to rate (1) the IAPS visual images as pleasant $(=0)$ or unpleasant $(=100),(2)$ the intensity of their emotional reactions when looking at the images (extremely mild $=0$; extremely intense $=100$ ), and (3) their willingness to look at the images (extremely willing $=0$; extremely unwilling $=100$ ), using three computer-presented sliding scales.

\section{Procedure}

The participants completed the experiment by working individually on a personal computer (software available from either the first or second author).

Practice tasks. Participants were presented with 12 trials during which they could choose whether to view a 'neutral' or 'nasty' (aversive) picture. The purpose of this phase was to familiarise the participants with the basic procedure and to allow them to view the types of neutral and aversive pictures that would be employed in the main body of the experiment. The data for practice tasks were not included in the analyses.

The participants were instructed to select one of two boxes that appeared on the screen that contained either the word nasty' or 'neutral'. A neutral or unpleasant IAPS image, as determined by the box selected, was presented on the screen for $6000 \mathrm{~ms}$. The participant was then instructed to hold the image in his or her head until the computer emitted a beep. The screen then remained blank for $2000 \mathrm{~ms}$ followed by the beep. The three sliding scales then appeared on the screen prompting the participant to make the subjective ratings in response to the image. When the participant had completed these questions and clicked on the button at the centre bottom of the screen the next trial started.

Phase 2: matching task. The procedure for this phase was similar to that used during the practice task but with an important difference: the participants were required to learn two separate sample-comparison relations. The comparison that a participant selected on any given trial determined whether an aversive or neutral visual image was presented. In the presence of one sample stimulus choosing a comparison that would produce an aversive visual image was defined as 'correct', whereas selecting the 'incorrect' comparison produced a neutral image. In the presence of a second sample stimulus choosing a comparison that produced a neutral visual image was defined as 'correct', whereas selecting the 'incorrect' comparison produced an aversive visual image. In principle, therefore, participants could avoid seeing the aversive images (once the two sample-comparison relations were learned), provided that they were willing to receive "incorrect" feedback across approximately half of the matching trials.

On the first trial an on-screen message instructed the participant to "Look at the box above and then choose one of the boxes below that goes with the one at the top". A sample stimulus then appeared in a rectangular box in the middle of the top half of the screen. The sample remained on the screen for $2000 \mathrm{~ms}$ and then two comparison stimuli appeared in separate rectangular boxes, one in the lower left corner and one in the lower right corner. The comparison stimuli remained on the screen until the participant clicked on a key ( $Z$ for left or $\mathrm{M}$ for right). If the participant chose the comparison defined as correct in the presence of the sample stimulus, the stimuli were removed from the screen and the word 'correct' appeared for $2000 \mathrm{~ms}$. If the participant clicked on the stimulus defined as wrong, the word 'wrong' appeared on the screen for $2000 \mathrm{~ms}$. The visual image, aversive or neutral, as determined by the comparison selected, then appeared on the screen for $6000 \mathrm{~ms}$. The participant was again instructed to hold the image in his or her head until the beep. The screen then remained blank for a further $2000 \mathrm{~ms}$ until the beep was presented. The three sliding scales appeared on the

\footnotetext{
${ }^{1}$ IAPS Slides. Neutral: 2190, 2200, 5500, 6150, 6570.5, 7000, 7002, 7006, 7009,7010, 7020, 7025, 7030, 7031, 7034, 7035, 7036, 7037, 7038, 7039, 7040, 7050, 7080, 7090, 7100, 7130, 7150, 7160, 7170, 7180, 7500, 7550, 7700. Aversive: 3080, 3101, 3110, 3120, 3140, 3168, 3170, $3181,3230,3250,3261,3266,3400,4664.2,6313,6315,6350,6550,6560,9008,9040,9252,9253,9265,9300,9301,9320,9400,9405,9410$, $9420,9432,9433,9584,9921$.
} 
screen, and when completed, the next trial started after $3000 \mathrm{~ms}$. A total of 36 trials were presented, irrespective of the performance of the participant. The two sample stimuli were presented in a quasi-random order ensuring that each stimulus was presented 18 times. The position of the two comparison stimuli was counterbalanced across trials. Immediately following the 36 trials the participants completed the postexperiment STAI-S questionnaire.

\section{Results and discussion}

Two female participants from the high-EA group withdrew from the experiment during the matching task and their data are not presented in this report. The remaining data was not normally distributed and thus nonparametric analyses were employed for Experiment 1 (this also applied to the behavioural and self-report data in Experiment 2). The results of these analyses are presented in Table 1.

Questionnaires. The high-EA group recorded higher levels of state anxiety than the low-EA group both before and after the experiment. The post-experiment scores of the high-EA group were significantly higher than the low-EA group. Additionally, only the high-EA group scores increased significantly from pre to postexperiment.

The two groups were similar with regard to personality traits as measured by the EPQ-R, apart from the dimension of neuroticism, with the high-EA group scoring significantly higher relative to the low-EA participants.

Table 1

Descriptive and inferential statistics for behavioural and self-report measures: Experiment 1

\begin{tabular}{|c|c|c|c|c|}
\hline & \multirow[t]{2}{*}{ Low-EA $(n=14)$} & \multirow[t]{2}{*}{ High-EA $(n=13)$} & \multicolumn{2}{|c|}{ Mann-Whitney } \\
\hline & & & $U$ & $p$ \\
\hline \multicolumn{5}{|l|}{ STAI-S } \\
\hline Pre- & $29(9)$ & $34.5(9)$ & & n.s. \\
\hline Post- & $33(7)$ & $39(14)$ & -2.083 & 0.0372 \\
\hline Wilcoxon $Z /$ Sig. & n.s. & $-2.480 / 0.00132$ & & \\
\hline \multicolumn{5}{|l|}{ EPQ-R } \\
\hline Neuroticism & $3(6)$ & $9(3.25)$ & -3.276 & 0.0011 \\
\hline \multicolumn{5}{|l|}{ Images viewed } \\
\hline Aversive & 17.5 & 17 & & n.s. \\
\hline Neutral & 18.5 & 19 & & n.s. \\
\hline Wilcoxon $Z /$ Sig. & n.s. & n.s. & & \\
\hline \multicolumn{5}{|l|}{ RT } \\
\hline Aversive & $2.181(0.496)$ & $2.504(1.463)$ & & n.s. \\
\hline Neutral & $1.888(1.252)$ & $1.877(0.845)$ & & n.s. \\
\hline Wilcoxon $Z /$ Sig & n.s. & $-2.621 / 0.0088$ & & \\
\hline \multicolumn{5}{|l|}{ Pleasantness } \\
\hline Aversive & $89.545(21.91)$ & $69.94(21.14)$ & -2.232 & 0.0256 \\
\hline Neutral & $12.05(20.76)$ & $21(21.182)$ & & n.s. \\
\hline Wilcoxon $Z$ / Sig & $-3.296 / 0.001$ & $-3.110 / 0.0019$ & & \\
\hline \multicolumn{5}{|l|}{ Emotional intense } \\
\hline Aversive & $84.42(25.14)$ & $53.68(40.877)$ & -2.135 & 0.0327 \\
\hline Neutral & $4.38(4.35)$ & $4.25(6.715)$ & & n.s. \\
\hline Wilcoxon $Z /$ Sig & $-3.296 / 0.001$ & $-3.110 / 0.0015$ & & \\
\hline \multicolumn{5}{|l|}{ Willing to look } \\
\hline Aversive & $63.915(31.5)$ & $68.19(36.208)$ & & n.s. \\
\hline Neutral & $4.675(4.1)$ & $10.39(29.39)$ & & n.s. \\
\hline Wilcoxon $Z /$ Sig & $-3.296 / 0.001$ & $-2.760 / 0.0058$ & & \\
\hline
\end{tabular}


Matching task. Compliance with the task as assessed by the correct response rate was similar for both groups, with a median of 28.5 for low-EA and 29 for high-EA out of a total of 36 trials. Additionally, both groups chose to see similar numbers of the aversive and neutral images.

Reaction times (RTs) for correct responses obtained during the two matching trial types were calculated in milliseconds (ms). Because the response on the first trial could not be based on prior learning (i.e. the participant could not know at this stage which stimulus produced the aversive or neutral picture), these RTs were excluded from the analyses. The data indicates that the high-EA group delayed significantly longer when emitting a response that produced an aversive relative to a neutral image; this significant difference was not observed for the low-EA group.

The low- and high-EA groups differentiated between the aversive and neutral images as indicated by their self-report ratings. Perhaps counter-intuitively, the low-EA group rated the aversive images as significantly more unpleasant and more emotionally arousing than did the high-EA participants.

Willingness to return. At the end of the experiment participants were asked if they were willing to participate in a second experiment. Seven participants from the high-EA group refused to participate further, in contrast to the low-EA group who all agreed to return. Similar issues regarding the retention of participants predisposed to avoidant coping styles have been reported in other studies (e.g., Eifert \& Heffner, 2003), indicating that unwillingness to continue participation provides a useful behavioural measure of avoidance.

\section{Experiment 2}

The second experiment replicated the matching task supplemented by the use of event-related potentials (ERPs) as a psycho-physiological measure that correlates with the processing of emotionally arousing stimuli. A recent study demonstrated that both pleasant and unpleasant pictures, taken from the IAPS, prompted a marked positive-going slow waveform, in contrast to the distinctly more negative slow-wave response to neutral pictures (Cuthbert, Schupp, Bradley, Birbaumer, \& Lang, 2000).

At the time of writing no published research had combined ERPs as a measure of emotional response with the investigation of avoidant coping styles. The use of ERPs permitted an exploratory analysis of the impact of emotionally valenced stimuli on participants predisposed towards high or low avoidance. The cerebral asymmetry of language function is well recognised, with the left relative to the right hemisphere more strongly implicated in verbal activity (Shtyrov, Pihko, \& Pulvermuller, 2005). Insofar as experiential avoidance is strongly linked to verbal responding (Hayes, Barnes-Holmes, \& Roches, 2001) it could be predicted that highEA individuals will show greater levels of left-hemisphere activity relative to low-EA individuals.

\section{Method}

\section{Participants}

Feldner et al. (2003) recently recommended the inclusion of participants from the continuum of experiential avoidance, and thus six participants were randomly selected from the mid range of the original screening AAQ. This resulted in three groups for Experiment 2: six participants from the low- and high-EA groups, and six participants with an AAQ score in the mid-range (mid-EA; mean $=41.2$ ). Each group were comprised of two males and four females.

\section{Measures}

The apparatus and stimuli used in Experiment 1 were also employed in Experiment 2 with the addition of ERPs recordings.

Electro-physiological measures. The EEG recordings were taken using a Brain Amp MR (Class IIa, Type BF) with approved control software (Brain Vision Recorder Version 1.0), and an approved electrode cap (BrainCap/BrainCap MR). The Brain Amp was controlled by a Dell personal computer with a Pentium 4 processor. The EEG data were analysed using approved analysis software (Brain Vision Analyser Version 
1.0). All of the hardware and software were manufactured and supplied by Brain Products GmbH, Munich, Germany.

\section{Procedure}

The experiment was conducted in a small, sound-attenuated, dimly lit room. Fifteen of the participants had completed Experiment 1, or a pilot study, some weeks before and were provided with a brief verbal reminder of the matching task. The three remaining participants from the mid-EA group (who were experimentally naïve) were first exposed to the Practice Task from Experiment 1. All participants were connected to the Brain Amp immediately before exposure to the matching task, identical to that employed in Experiment 1 . They were instructed to avoid unnecessary head and body movements during the presentation of pictures on the computer screen. When the matching task was complete, the EEG recording was discontinued and the electrodes were removed. The participants then completed the post-experiment STAI-S.

Recordings. Evoked potentials were recorded and analysed from nine, sintered AG/AG-CI scalp electrodes positioned over the right and left hemispheres at the frontal (F3, Fz \& F4), central (C3, Cz \& C4) and parietal (P3, Pz \& P4) sites, as employed by Cuthbert et al. (2000). Site FCz was used as reference and the vertex as ground. In accordance with Cuthbert et al., the amplifier resolution was $0.1 \mu \mathrm{V}$ and the high-frequency cut-off was $35 \mathrm{~Hz}$. The sampling rate was $1250 \mathrm{~Hz}$, decimated off-line to a $125 \mathrm{~Hz}$ sampling rate. The EEG was collected continuously and edited off-line. Two additional electrodes monitored any horizontal and vertical eye movement. Average ERPs were calculated across all exposures to the aversive and neutral stimuli. Evoked potentials were not separated out in terms of correct and incorrect matching trials because the primary focus was on the participants' slow-wave (6s) ERP to the emotional content of the stimuli.

\section{Results and discussion}

The descriptive and inferential statistics for the behavioural and self-report data are presented in Table 2.

Questionnaires. The three groups scored similar levels of state anxiety prior to the experiment with the highand mid-EA groups increasing post-experiment, but there were no statistically significant differences. The three groups were relatively similar with regard to personality traits as measured by the EPQ-R. As in Experiment 1, the high-EA group scored significantly higher on the neuroticism subscale than both the lowEA and mid-EA group.

Matching task. The correct responses made during the 36 matching trials were calculated for the three groups (median: high-EA $=24$, mid-EA $=30.5$, low-EA $=27$ ). There was no difference across the groups in terms of accuracy or for the number of aversive or neutral images viewed.

Overall, the high-EA group produced significantly longer RTs for both aversive and neutral images than the other two groups, but critically, as in Experiment 1, the high-EA group also produced significantly longer RTs for aversive versus neutral images.

The high, mid- and low-EA groups differentiated between the aversive and neutral images as demonstrated by their self-report ratings. Paradoxically, as in Experiment 1, the low-EA group rated the aversive images as more unpleasant (significantly) and more emotionally arousing (but not significantly) than did the high-EA group.

ERPs measures. In general, the filtering and other noise-reducing techniques that Cuthbert et al. (2000) employed were also applied to the current EEG data. First, the continuous EEG signals for each participant were filtered (frequency $0.0159155 \mathrm{~Hz}$, time constant $10 \mathrm{~s}$ ), and then segmented for neutral and aversive pictures. The segments were divided into $6120 \mathrm{~ms}$ epochs commencing $120 \mathrm{~ms}$ before picture onset. Vertical and horizontal ocular artefacts were then corrected, and any segments on which EEG or electro-ocular activity exceeded $\pm 75 \mu \mathrm{V}$ were rejected. If more than 12 of 36 segments obtained from a single participant contained an artefact, all of the data for that participant were removed from the analysis. The data for nine participants were thus rejected (leaving 4 high, 1 mid- and 4 low-EA participants). The segments for the remaining nine 
Table 2

Descriptive and inferential statistics for behavioural and self-report measures: Experiment 2

\begin{tabular}{|c|c|c|c|c|c|}
\hline & \multirow{2}{*}{ Low-EA $(n=6)$} & \multirow[t]{2}{*}{ Mid-EA $(n=6)$} & \multirow[t]{2}{*}{ High-EA $(n=6)$} & \multicolumn{2}{|c|}{ Kruskall-Wallis } \\
\hline & & & & $H$ & $p$ \\
\hline \multicolumn{6}{|l|}{ STAI-S } \\
\hline Pre- & $26.5(6)$ & $27.5(15)$ & $28(19)$ & & n.s. \\
\hline Post- & $24.5(11)$ & $43(33)$ & $38(15)$ & & n.s. \\
\hline Wilcoxon $Z / \operatorname{Sig}$ & n.s. & n.s. & n.s. & & \\
\hline \multicolumn{6}{|l|}{ EPQ-R } \\
\hline Neuroticism & $4(4)$ & $2(7)$ & $11(3)$ & 10.152 & 0.0062 \\
\hline \multicolumn{6}{|l|}{ Images viewed } \\
\hline Aversive & $18.5(6)$ & $17(4)$ & $18(22)$ & & n.s. \\
\hline Neutral & $17.5(6)$ & $19(4)$ & $18(12.75)$ & & n.s. \\
\hline Wilcoxon $Z /$ Sig & n.s. & n.s. & n.s. & & \\
\hline \multicolumn{6}{|l|}{ RT } \\
\hline Aversive & $0.812(0.113)$ & $0.715(1.387)$ & $3.315(0.798)$ & 7.895 & 0.0193 \\
\hline Neutral & $1.005(0.537)$ & $0.786(0.0737)$ & $1.426(0.498)$ & 6.4 & 0.0408 \\
\hline Wilcoxon $Z /$ Sig & n.s. & n.s. & $-2.023 / 0.0431$ & & \\
\hline \multicolumn{6}{|l|}{ Pleasantness } \\
\hline Aversive & $84(12.29)$ & $93.96(8.91)$ & $67.82(14.83)$ & 6.503 & 0.0408 \\
\hline Neutral & $24(38.06)$ & $31.32(16.68)$ & $15.17(16.102)$ & & n.s. \\
\hline Wilcoxon $Z /$ Sig & $-2.201 / 0.0277$ & $-2.201 / 0.0277$ & $-2.023 / 0.0431$ & & \\
\hline \multicolumn{6}{|l|}{ Emotional intense } \\
\hline Aversive & $80.67(12.53)$ & $85.92(18.63)$ & $55.89(20.25)$ & & n.s. \\
\hline Neutral & $3.5(3.68)$ & $5.72(9.84)$ & $3.94(10.46)$ & & n.s. \\
\hline Wilcoxon $Z /$ Sig & $-3.296 / 0.001$ & $-2.201 / 0.0277$ & $-3.110 / 0.0015$ & & \\
\hline \multicolumn{6}{|l|}{ Willing to look } \\
\hline Aversive & $55.72(12.95)$ & $67.8(22.83)$ & 68.55 (10.94) & & n.s. \\
\hline Neutral & $5.77(32.98)$ & $17.12(20.73)$ & $16.67(56.74)$ & & n.s. \\
\hline Wilcoxon $Z / \operatorname{Sig}$ & n.s. & $-1.992 / 0.0464$ & n.s. & & \\
\hline
\end{tabular}

participants were then baseline corrected (using the $120 \mathrm{~ms}$ pre-stimulus interval), and finally averaged for neutral and aversive pictures.

The grand average waveforms, calculated across all participants, for each of the nine electrode sites (F3, Fz, F4, C3, Cz, C4, P3, Pz \& P4) for the 6-s presentation of aversive and neutral stimuli are presented in Fig. 1. Visual inspection of these waveforms indicates that from around $500 \mathrm{~ms}$ post-stimulus onset, the waveforms for the aversive stimuli (black lines) show greater positivity than for neutral stimuli (grey lines). To compare the ERPs data for the high- with the low/mid-EA groups, Table 3 presents the average area dimensions $(\mu \mathrm{V} \mathrm{ms})$ for the left, middle, and right sites (collapsed across anterior/posterior positions). The high-EA group produced greater negativity relative to the low/mid-EA group, for both picture types. A large negative ERP response to the aversive images was produced over the left hemisphere by the high-EA group, which contrasted with the positive activations observed for the low/mid-EA group.

Although the $\mathrm{n}$ was very low, given the lack of currently available ERP data in this research area it was deemed useful to conduct statistical analyses. The area dimensions $(\mu \mathrm{Vms})$ were subjected to a four-way $2 \times 2 \times 3 \times 3$ mixed ANOVA with high versus low/mid as a between participant factor, and image type (aversive versus neutral), electrode position (anterior, midline, posterior) and laterality (left, middle, right) as repeated measures factors. The ANOVA revealed two main effects, for picture content $[F(1,1)=53.837$, $p=0.0002$, partial eta squared $=0.43]$ and laterality $[F(2,1)=8.247, p=0.0043$, partial eta squared $=0.54]$. A 3-way interaction was also identified for EA group by laterality by anterior-posterior position $[F(4,8)=3.950, p=0.0115$, partial eta-squared $=0.36]$. No other interaction effects were identified. 


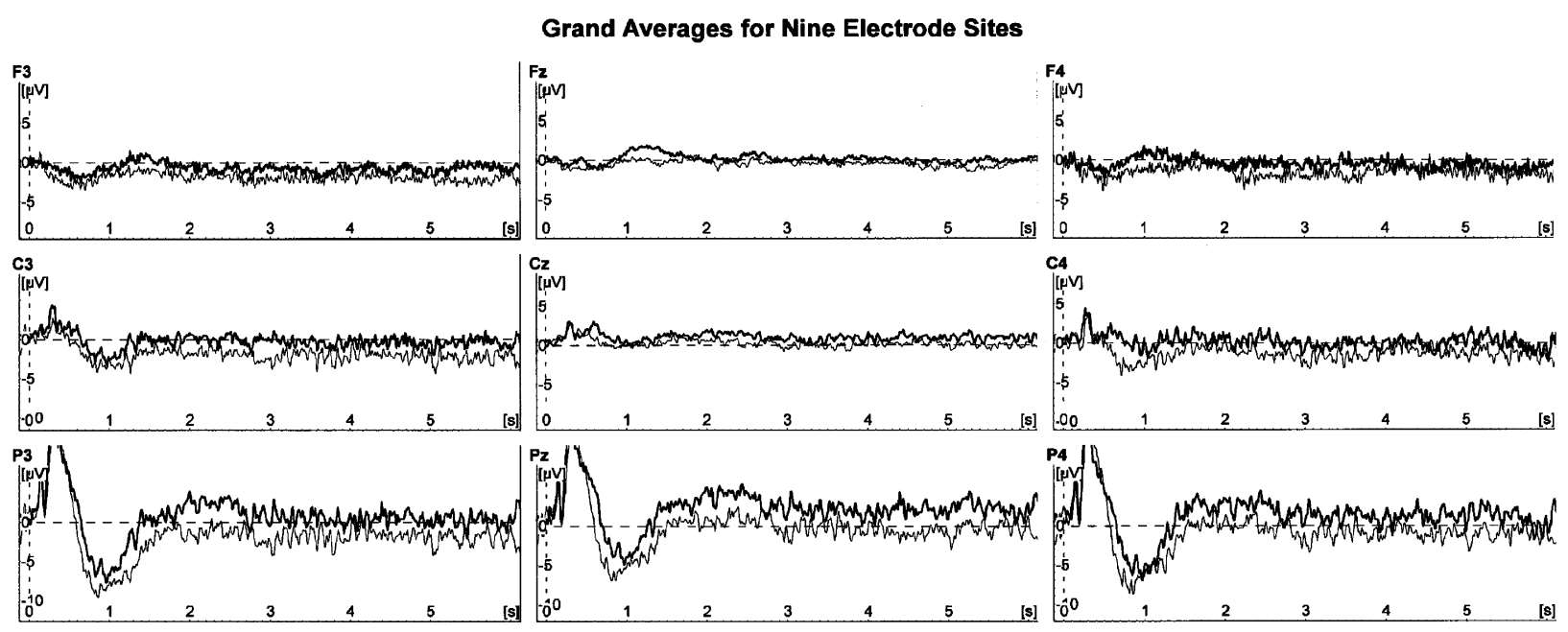

Fig. 1. Grand average waveforms calculated across nine participants for aversive (black lines) and neutral images (grey lines).

Table 3

Average area dimensions $(\mu \mathrm{Vms})$ for aversive and neutral images across left, middle and right electrode sites $(+\mathrm{SE})$

\begin{tabular}{lcc}
\hline & Low/mid-EA $(n=5)$ & High-EA $(n=4)$ \\
\hline Left & & \\
$\quad$ Neutral & $-5657(2478)$ & $-15758(2488)$ \\
Aversive & $2558(2543)$ & $-5289(2488)$ \\
Middle & & $-2212(2092)$ \\
$\quad$ Neutral & $-171(2498)$ & $3377(1917)$ \\
Aversive & $7394(3480)$ & \\
Right & & $-7758(2752)$ \\
$\quad$ Neutral & $-5397(2711)$ & $-390(1683)$ \\
Aversive & $2793(2766)$ & \\
\hline
\end{tabular}

In order to investigate the interaction effect, two separate repeated measures ANOVAs were conducted for the two groups (high-EA versus low/mid-EA). For the high-EA group, the ANOVA revealed a significant main effect for image type $[F(1,3)=30.565, p=0.0117$, partial eta-squared $=0.91]$ and laterality $[F(2,6)=10.832, p=0.0102$, partial eta-squared $=0.78]$. There were no significant interaction effects. Sheffe post-hoc tests revealed significant differences between left and middle electrode positions $(p=0.0104)$, but not between left and right $(p=0.0930)$ or middle and right $(p=0.2312)$. The ANOVA for the combined low $/$ midEA group revealed a significant main effect for image type $[F(1,4)=26.5, p=0.0068$, partial etasquared $=0.86$ ] but not for laterality, and no interaction effects.

Overall, the ERPs measures indicated that both the high-EA and combined low/mid-EA groups reliably discriminated between image types, with the aversive stimuli evoking more positivity relative to neutral images. Only the high-EA group showed significant differences for laterality, with electrodes over the left hemisphere producing greater negative activity relative to those in the midline.

\section{General discussion}

Consistent with experimental predictions, the reaction time measure but not image-selected discriminated between high- and low/mid-EA groups. Specifically, the high-EA groups took significantly longer to emit a correct response that produced an aversive relative to a neutral image. Conversely, the reaction times of the 
low- and mid-EA groups did not differ significantly between aversive and neutral stimuli. Insofar as a response delay (that produces an aversive stimulus) may be interpreted as a measure of avoidance, the current data provide support for the construct of experiential avoidance. Although the ' $n$ ' was relatively small, particularly in the second experiment, similar effects were found in each experiment thus supporting the reliability of the findings. In any case, these data appear to be consistent with previous research. For example, a recent study used a $\mathrm{CO}_{2}$ challenge to compare the effects of acceptance versus control interventions on avoidance of panicrelated symptoms with participants who scored high on anxiety sensitivity (Eifert \& Heffner, 2003). Participants trained in a control-based strategy (training in diaphragmatic breathing) took progressively longer to initiate each trial relative to those in the acceptance condition (instructed to mindfully observe). The authors concluded that the control-based intervention group used delay as a means of controlling their symptoms. In the current study, taking longer to emit a response that produced an aversive image may have reflected a similar delaying strategy.

In Experiment 1, pre-experiment state anxiety scores did not discriminate between the groups, yet the highEA group reported higher levels of anxiety following the experiment relative to the low-EA group. This result is consistent with the findings of Feldner et al. (2003) who found no differences in anticipatory anxiety but greater levels of post-experiment anxiety amongst those high versus those low in experiential avoidance. Furthermore, the fact that the high-EA group in the current study reported greater anxiety levels is consistent with a correlation between anxiety and both the AAQ and the Cognitive-Behavioral Avoidance Scale (Ottenbreit \& Dobson, 2004).

Although, the high-EA groups in the current study reported higher levels of anxiety they rated the aversive images as being less unpleasant and less emotionally arousing than did the low/mid-EA groups. In contrast, previous studies using a $\mathrm{CO}_{2}$ challenge have found that high- compared to low-avoiders report greater levels of cognitive distress (Feldner et al., 2003). On balance, a $\mathrm{CO}_{2}$ challenge constitutes a very different stimulus from that of aversive visual material, and thus different outcomes are not entirely surprising. Nonetheless, Sloan (2004) found that high avoiders reported greater negative affect to unpleasant film clips compared to low avoiders (Sloan, 2004). Perhaps the lower ratings given to the aversive images by the high-EA group in the current study constituted an attempt to suppress the unpleasant content while continuing to view the material, a strategy that may not have emerged across only two film clips in the Sloan study. If this is the case, it is possible that the higher levels of post-experiment anxiety reported by the high- relative to the low-EA groups reflects a post-suppression 'rebound' effect, with a paradoxical increase in anxiety, consistent with the findings reported in the thought suppression literature (Wenzlaff \& Wegner, 2000).

The ERP measures indicated that both the high-EA and low/mid-EA groups reliably discriminated between image-types, with the aversive stimuli evoking more positivity relative to neutral images. Furthermore, the ERPs measure suggested a neurophysiological difference between the high and low/mid avoiders. That is, only the high-EA group showed greater negativity for the electrodes recording over the left hemisphere relative to those in the midline. The asymmetry of cerebral function is complex and known to be influenced by factors such as emotional processes, handedness and gender (Rodway, Wright, \& Hardie, 2003). The latter two factors were not controlled for in the current study with the small number of participants whose ERP data were suitable for analysis. Nevertheless, insofar as verbal activity typically creates greater activation in the left hemisphere (Shtyrov et al., 2005) the current findings could indicate greater verbal activity for the high-EA group (see Borkovec, Ray, \& Stober, 1998). Because statistical analyses indicated that the laterality effect in the current study did not differentiate between aversive and neutral images, the data may reflect a generalised use of verbal avoidance strategies in the context of an emotionally challenging experimental environment. Future research could explore this issue.

\section{Acknowledgements}

The preparation of this article was supported by the Irish Research Council for the Humanities and the Social Sciences, through a Government of Ireland Scholarship. Our thanks go to Steve Hayes and Denise Sloan for their helpful comments on an earlier draft. 


\section{References}

Borkovec, T. D., Ray, W. J., \& Stober, J. (1998). Worry: A cognitive phenomenon intimately linked to affective, physiological and interpersonal behavioural processes. Cognitive Therapy and Research, 22(6), 561-576.

Cuthbert, B. N., Schupp, H. T., Bradley, M. M., Birbaumer, N., \& Lang, P. J. (2000). Brain potentials in affective picture processing: Covariation with autonomic arousal and affective report. Biological Psychology, 52, 95-111.

Eifert, G. H., \& Heffner, M. (2003). The effects of acceptance versus control contexts on avoidance of panic-related symptoms. Journal of Behavior Therapy and Experimental Psychiatry, 34(3-4), 293-312.

Eysenck, H. J., \& Eysenck, S. B. G. (1991). Manual of the Eysenck personality questionnaire. London: Hodder \& Stoughton.

Feldner, M. T., Hekmat, H., Zvolensky, M. J., Vowles, K. E., Secrist, Z., \& Leen-Feldner, E. W. (2006). The role of experiential avoidance in acute pain tolerance: A laboratory test. Journal of Behavior Therapy and Experimental Psychiatry, 37(2), 146-158.

Feldner, M. T., Zvolensky, M. J., Eifert, G. H., \& Spira, A. P. (2003). Emotional avoidance: An experimental test of individual differences and response suppression using biological challenge. Behaviour Research and Therapy, 41, 403-411.

Hayes, S. C., Barnes-Holmes, D., \& Roches, B. (Eds.). (2001). Relational Frame Theory: A post-Skinnerian account of human language and cognition. New York: Kluwer Academic.

Hayes, S. C., Strosahl, K. D., \& Wilson, K. G. (1999). Acceptance and commitment therapy: An experiential approach to behaviour change. New York: Springer.

Hayes, S. C., Strosahl, K. D., Wilson, K. G., Bisset, R. T., Pistorello, J., Toarmino, D., et al. (2004). Measuring experiential avoidance: A preliminary test of a working model. The Psychological Record, 54, 553-578.

Lang, P. J., Bradley, M. M., \& Cuthbert, B. N. (1999). International affective picture system (IAPS): Instruction manual and affective ratings. Technical report A-4, The Center for Research in Psychophysiology, University of Florida.

McAuliffe, D. (2004). Rule following and depressive symptomology in an adolescent population. Unpublished doctoral dissertation. National University of Ireland Maynooth, Co., Kildare.

Ottenbreit, N. D., \& Dobson, K. S. (2004). Avoidance and depression: The construction of the Cognitive-Behavioral Avoidance Scale. Behaviour Research and Therapy, 42, 293-313.

Rodway, P., Wright, L., \& Hardie, S. (2003). The valence-specific laterality effect in free viewing conditions: The influence of sex, handedness and response bias. Brain and Cognition, 53, 452-463.

Shtyrov, Y., Pihko, E., \& Pulvermuller, F. (2005). Determinants of dominance: Is language laterality explained by physical or linguistic features of speech? NeuroImage, 27, 37-47.

Sloan, D. M. (2004). Emotion regulation in action: Emotional reactivity in experiential avoidance. Behaviour Research and Therapy, 42(11), 1257-1270.

Spielberger, C. D. (1983). State-trait anxiety inventory (Form Y). Redwood City, CA: Mind Garden.

Van Hermert, D. A., van de Vijver, F. J. R., Poortinga, Y. A., \& Georgas, J. (2002). Structural and functional equivalence of the Eysenck Personality Questionnaire within and between countries. Personality and Individual Differences, 33, 1229-1249.

Wenzlaff, R. M., \& Wegner, D. M. (2000). Thought suppression. Annual Review of Psychology, 51, 59-91. 